\title{
Prescribing pattern and utilization of selected antimicrobials in a tertiary hospital of Nepal
}

\author{
Upasana Acharya ${ }^{1}$, PharmD; Ravi Mahat ${ }^{2}$, FCPS; Subhash Prasad Acharya ${ }^{3}$, MD, FCCP; Bishnu \\ Pahari, ${ }^{4}$, DM; Prashant Tripathi ${ }^{5}$, MD \\ ${ }^{1}$ Clinical Pharmacist, Grande International Hospital, Kathmandu, Nepal \\ ${ }^{2}$ Department of Pulmonary Medicine, Grande International Hospital, Kathmandu, Nepal \\ ${ }^{3}$ Department of Critical Care Medicine, Grande International Hospital, Kathmandu, Nepal \\ ${ }^{4}$ Department of Nephrology and Transplant Medicine, Grande International Hospital, Kathmandu, Nepal \\ ${ }^{5}$ Department of Internal Medicine, Grande International Hospital, Kathmandu, Nepal
}

\section{Corresponding author}

Upasana Acharya, PharmD

Email: acharya.upa@gmail.com

Received 23 Mar 2019

Accepted 19 Aug 2019

Introduction:

This study was done to understand the purpose of using higher grade antimicrobials in a one year period and to compare the consumption of commonly prescribed parenteral antimicrobial agents in four consecutive years in a tertiary care hospital of Nepal.

\section{Materials \& Methods:}

A retrospective observational study was carried out to collect the information on prescription of selected six classes of antimicrobial for all admitted patients aged 2 years or above for a one-year period. Further, the antimicrobial consumption tool 2016 was used to calculate defined daily dose (DDD) per 100 bed days of commonly dispensed parenteral antimicrobials for four consecutive years.

\section{Results:}

A total of 2568 antimicrobial supply forms prescribing higher grades antimicrobials were analyzed. The indications for use of antimicrobials were mainly for empiric treatment. Among the infections being treated, lower respiratory tract infection (LRTI) was the most frequent cause. Piperacillintazobactam was most frequently prescribed followed by Meropenem. Also, DDD for commonly used parenteral antimicrobials showed increasing trends over four consecutive years.

\section{Conclusion:}

The prescription of higher grades of antimicrobial for empiric treatment and surgical prophylaxis need to be monitored. The rate of consumption of such antimicrobials could not be assessed due to lack of similar studies but the increase in DDD/100 bed days each year suggested the rise in consumption of antimicrobial in the same institution.

Key words: Antimicrobials; Nepal; Defined daily dose; Indication 


\section{Introduction}

Antibiotics are among the most commonly prescribed drugs in hospitals and around $30 \%$ of the hospitalized patients are treated with antibiotics ${ }^{1,2}$. Collecting data on motives for antibiotic use is the first step in managing the problem of antibiotic resistance as such information is necessary for a constructive approach to problems that arise from the multiple antibiotics prescription ${ }^{3,4,5,6}$.

The Anatomical Therapeutic Chemical (ATC) classification system and the Defined Daily Dose (DDD) as a measuring unit have become the gold standard for international drug utilization research ${ }^{7,8}$. Few studies have been carried out in western and eastern regions of Nepal ${ }^{9,10,11,12}$ with regard to drug utilization in hospitals, whereas such information from central Nepal, moreover from a tertiary referral center is lacking. The main objective of this study was to understand the prescribing patterns of higher grade antimicrobials and to measure the consumption of antimicrobial agents in the same hospital for four consecutive years.

\section{Methods}

One year retrospective study was carried out at Grande International Hospital, a 200 bedded tertiary hospital in Kathmandu, Nepal from October 2016 to September 2017 to understand the indications of prescribing selected higher grade anti-microbials. The occupancy rate of hospital at the time of study was 0.6 with eleven beds in Intensive Care Unit. The reason for selecting this particular time period is due to availability of data entered in system only for this time frame.

A pre designed antimicrobial ordering form was used to collect the information such as patient demographics, department, date of starting antibiotics, duration, name of the agent, indication for use and infection being treated. Only selected higher grades antimicrobials (Carbapenem, Aminoglycosides, Glycopeptides, Tigecycline, Beta lactam in combination with beta lactamase inhibitors, Polymyxins) were included in the study as restricted antimicrobial forms need to be filled by the medical officer if the primary consultant decides to prescribe those antibiotics on the basis of local antimicrobial guidelines and send that form to pharmacy to get the supply The antibiotics would be supplied through the form for a maximum of seven days and if days of therapy needed to be extended, justification for such use was to be provided in the form and approval from the antimicrobial committee was a must who decide if the request was compliant with our guidelines. Those collected information were entered and analyzed using MS Excel 2007.

Secondly, DDDs were calculated separately for commonly prescribed antibiotics (including those supplied through restricted antimicrobial form, total 26 parenteral antimicrobial agents were taken based on volume of sales from Pharmacy) using AMC tool 2016 for four consecutive years i.e. 2013 to 2017 A.D, which gives the DDD for the anti-microbial that has been assigned ATC code for each route when volume of consumption, strength and pack size are entered. This hospital follows the DDD as defined by WHO. The DDD value is updated periodically ${ }^{13}$. The DDD is converted to DDD/100 bed days using occupancy rate. The formula employed by the system is:

DDD/100 bed days $=\frac{\text { No.of grams of antibiotic used } \times 100}{\text { WHO DDD Units }(g) \times \text { No.of bed days }}$

The DDD/100 bed days was calculated for commonly used antibiotics in the hospital for four years to compare the consumption of antimicrobials in different years.

\section{Results}

A total of 2568 restricted antimicrobial supply forms were included. Ten higher grades antimicrobials Piperacillin-tazobactam, Meropenem, Imipenem, Amikacin, Gentamycin, Vancomycin, Teicoplanin, Tigecycline, Polymyxin (B\&E) were analyzed which showed that forms filled to request Piperacillintazobactam were highest followed by Meropenem as shown in Figure 1.

On further analysis, the indication for use of antimicrobials was mainly for empiric treatment.

Figure 2 shows the different indications for request of higher grade antimicrobials.

Among the infections being treated, Lower respiratory tract infection was the leading cause (43.3\%), then sepsis (12.2\%), UTI (6.4\%) and cellulites $(5.9 \%)$.

The consumption of higher grades antimicrobials were high in the intensive care, high dependency care, pediatric care and cardiac care units as shown in figure 3. 


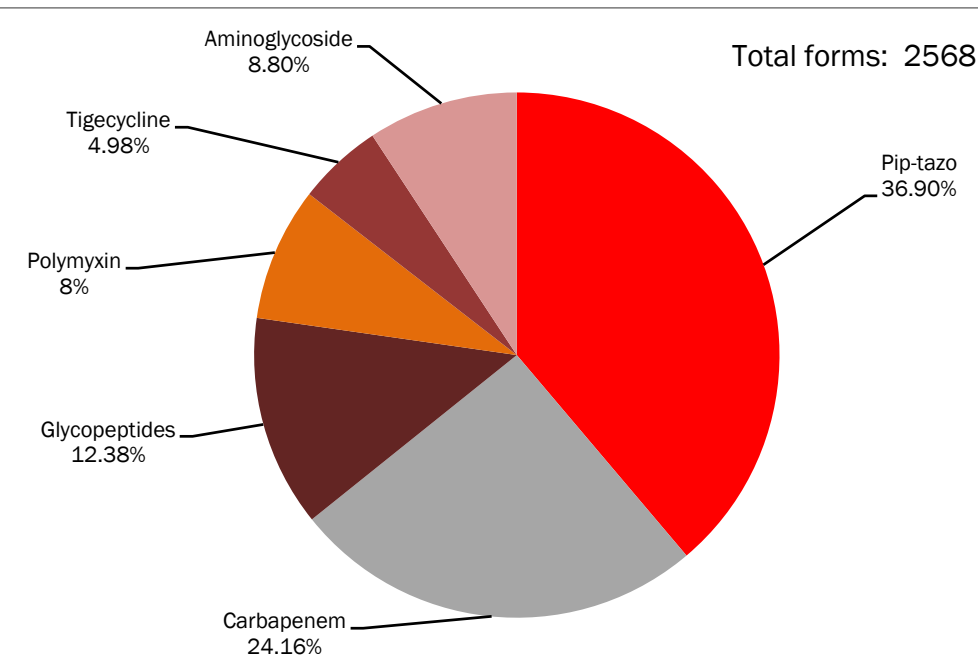

Figure 1 - Percentages showing patients who were prescribed restricted antimicrobials in 2017

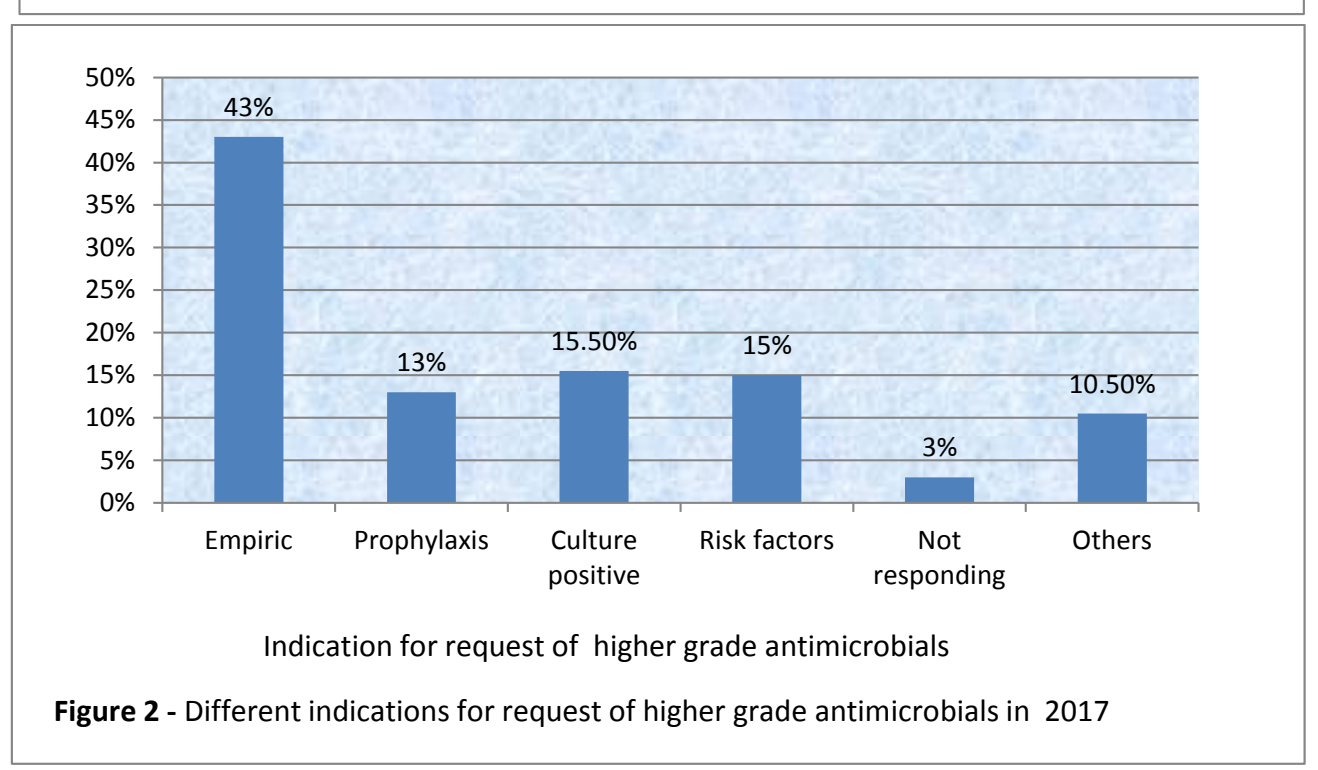

Figure 2 - Different indications for request of higher grade antimicrobials in 2017

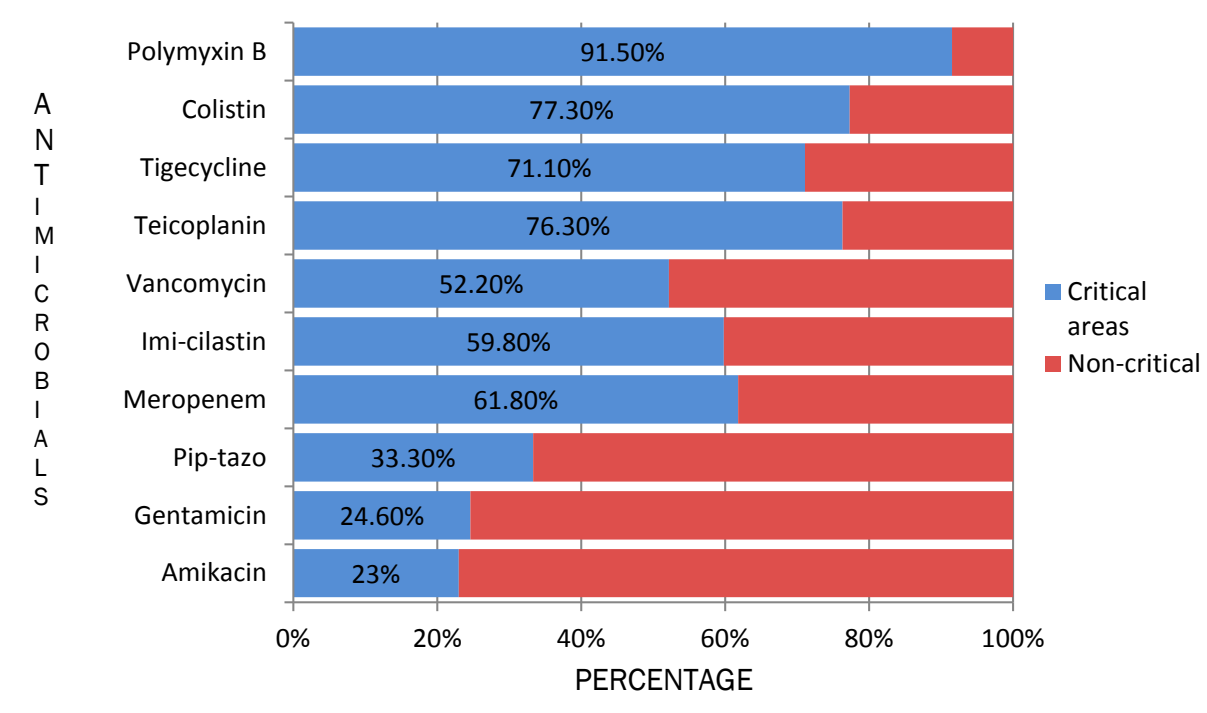

Figure 3 - Perentages showing consumption of higher grade antimicrobials in critical and non critical areas in hospital 


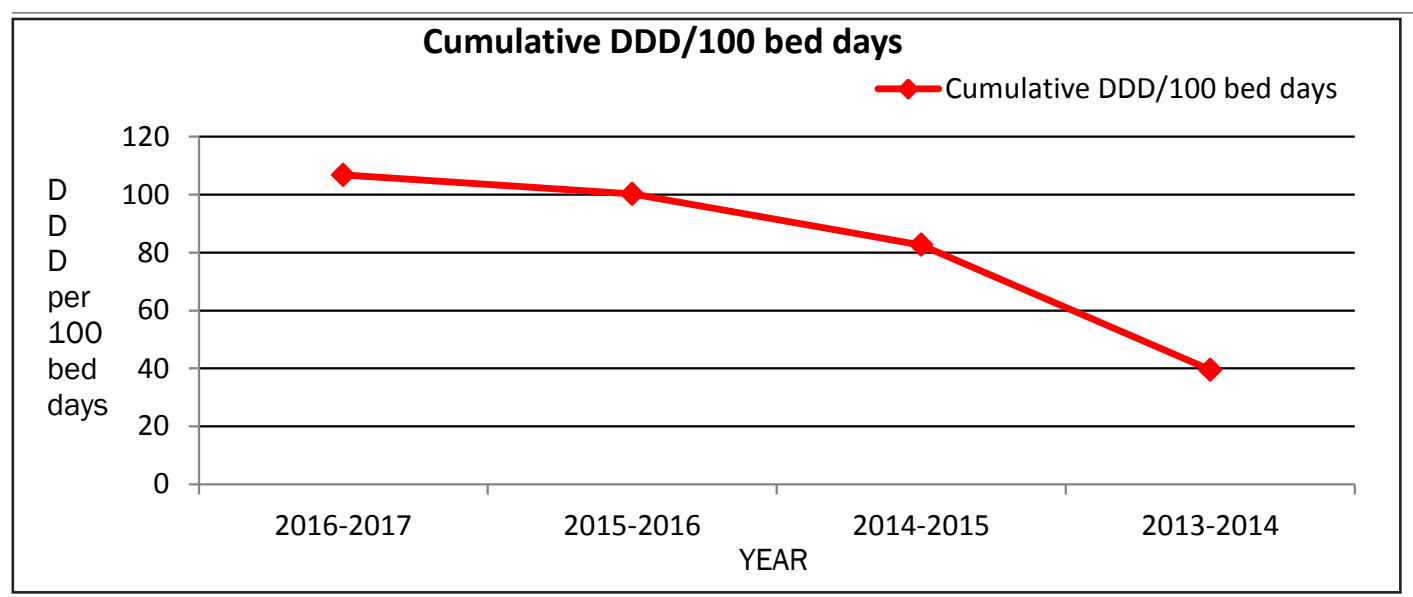

Figure 4 - Line graph showing cumulative defined daily dose per 100 bed days in four consecutive years

The total DDD is on an increasing trend over the years indicating markedly raised antimicrobials consumption over the four years.

Ceftriaxone is the most commonly prescribed in all years followed by meropenem, gentamicin and piperacillin-tazobactam (Table 1). The consumption of antimicrobials such as piperacillin-tazobactam, vancomycin, teicoplanin, ceftriaxone, amoxicillinclavulanic acid, metronidazole were seen to be low in 2017 compared to the year 2016.

Table 1. Defined Daily Dose (DDD) of commonly dispensed parenteral antimicrobials in four consecutive years

DDD/100 BED DAYS

\begin{tabular}{|c|c|c|c|c|c|c|}
\hline \multirow{2}{*}{ ANTIMICROBIAL AGENT } & \multirow{2}{*}{ STRENGTH } & \multirow{2}{*}{ ATC CODE } & \multicolumn{4}{|c|}{ YEAR } \\
\hline & & & $2016 / 2017$ & $2015 / 2016$ & $2014 / 2015$ & $2013 / 2014$ \\
\hline Ceftriaxone & $0.5 \mathrm{~g} / 1 \mathrm{~g}$ & J01DD04 & 18.21 & 18.8 & 15.34 & 9.34 \\
\hline Meropenem & $0.5 \mathrm{~g} / 1 \mathrm{~g}$ & J01DH02 & 15.02 & 11.34 & 3.6 & 1.72 \\
\hline Gentamycin & $0.8 \mathrm{~g}$ & J01GB03 & 13.31 & 10.72 & 10.1 & 5.33 \\
\hline Metronidazole & $0.5 \mathrm{~g}$ & J01XD01 & 9.3 & 11.9 & 8.3 & 5.3 \\
\hline Piperacillin-tazobactam & $2.25 \mathrm{~g} / 4.5 \mathrm{~g}$ & J01CR05 & 9.29 & 10.32 & 8.34 & 4.1 \\
\hline Cefazolin & $0.5 \mathrm{~g} / 1 \mathrm{~g}$ & J01DB04 & 8.41 & 7.49 & 6.78 & 1.2 \\
\hline Colistin & $1 \mathrm{mu} / 2 \mathrm{mu}$ & J01XB01 & 8.31 & 6.2 & 2.16 & 0.38 \\
\hline Levofloxacin & $0.5 \mathrm{~g}$ & J01MA12 & 4.3 & 2.98 & 2.25 & 3.25 \\
\hline Ciprofloxacin & $0.2 \mathrm{~g}$ & J01MA02 & 1.13 & 1.23 & 1.23 & 0.62 \\
\hline Amoxicillin-clavulanic acid & $1.2 \mathrm{~g}$ & J01CR02 & 4.2 & 4.4 & 4.45 & 2.02 \\
\hline Teicoplanin & $0.2 \mathrm{~g} / 0.4 \mathrm{~g}$ & J01XA02 & 3.3 & 4.7 & 2.63 & 0.97 \\
\hline Ampicillin+sulbactam & $1.5 \mathrm{~g}$ & J01CR01 & 3.03 & 0.004 & 0 & 0 \\
\hline Azithromycin & $0.5 \mathrm{~g}$ & J01FA10 & 2.78 & 2.7 & 2.3 & 0.64 \\
\hline Tigecycline & 0.05 & J01AA12 & 2.53 & 0.92 & 1.1 & 0 \\
\hline Vancomycin & 0.5 & J01XA01 & 2.03 & 2.26 & 2 & 0.65 \\
\hline Amikacin & $0.5 \mathrm{~g}$ & J01GB06 & 1.9 & 1.8 & 3.8 & 2.86 \\
\hline Imipenem+cilastin & $0.5 \mathrm{~g}$ & J01DH51 & 1.6 & 0.67 & 2.8 & 0 \\
\hline Flucloxacillin & $0.5 \mathrm{~g}$ & J01CF05 & 1.57 & 1.25 & 1.047 & 0.3 \\
\hline Polymyxin B & $0.05 \mathrm{~g}$ & J01XB02 & 0.83 & 0 & 0 & 0 \\
\hline Cloxacillin & $0.5 \mathrm{~g}$ & J01CF02 & 0.8 & 2.04 & 1.076 & 0.52 \\
\hline Tobramycin & $0.08 \mathrm{~g}$ & J01GB01 & 0.6 & 0.4 & 1.3 & 0.52 \\
\hline Clindamycin & $0.3 \mathrm{~g} / 0.6 \mathrm{~g}$ & J01FF01 & 0.73 & 0.46 & 0.66 & 0.21 \\
\hline Ceftazidime & $1 g$ & J01DD02 & 0.32 & 0.09 & 0.3 & 0.1 \\
\hline TOTAL (DDD/ 100 BED DAYS) & & & 113.7 & 102.8 & 84 & 40.12 \\
\hline
\end{tabular}




\section{Discussion}

Our findings of respiratory tract infections accounting formaximumuse of higherantimicrobials are similar to the findings of other studies. The European surveillance is a point prevalence study which included 20 European hospitals only for two calendar weeks ${ }^{14}$ whereas studies done by Dannis et al. and Shankar et al. in a University hospital of Netherland and Nepal respectively only included patients admitted in ICU (15 beds in Netherland/10 beds in Nepal) ${ }^{14,4,9}$. In these studies, patients getting any antimicrobials during their hospital stay were included whereas our study has only focused on patients getting higher grade antimicrobials in various departments of the hospital

Even though the DDD for Carbapenem was higher than Piperacillin-Tazobactam in the year 2016/2017, the prescription analysis showed Piperacillintazobactam to be commonly prescribed among the restricted classes of antimicrobials Carbapenems even thoug prescribed to fewer patients was found to be continued for a longer duration. The total DDD/100 bed days in 2016/2017 is similar to the result of study conducted in one of the hospital in Nepal by Shanker et al. ${ }^{10}$ which included patients in the 60 bedded internal medicine ward for a period of 3 months. But our study includes the consumption of entire hospital for one year. We can hence conclude that DDD/100 bed days are low in our study when compared to the study done by Shanker et al.

Ceftriaxone is the most common antimicrobial to be prescribed which is similar to studies carried out in Nepal and Indonesia ${ }^{11,15}$ but higher compared to studies done in intensive care unit and internal medicine department of the same hospital on year 2003 and 2005 which was only 7.9 and $4.56 \mathrm{DDD} / 100$ bed days respectively ${ }^{9,10}$. In a study carried out in Nepal (2008) and Indonesia (2015), DDD of ceftriaxone had not been calculated so direct comparison with our study could not be done. A similar study conducted in Brazil also analyzed the selected antimicrobials over a long time period in an entire hospital except one unit and concluded that the use of ceftriaxone was high $^{16}$ - it had increased from 2.57 DDD/100 bed days (1999 AD) to $8.32 \mathrm{DDD} / 100$ bed days (2004 $A D$ ). In our case it has raised from $9.34 \mathrm{DDD} / 100$ bed days (2014 AD) to $18.21 \mathrm{DDD} / 100$ bed days (2017 AD). The use of ceftriaxone in a study done in Bulgaria has only reached $5.61 \mathrm{DDD} / 100$ bed days which is three folds lower than our value and that too included entire hospital and selected antimicrobials ${ }^{17}$. A point prevalence study in a 372 bedded hospital in Turkey showed highest consumption of third generation cephalosporin with $35 \mathrm{DDD} / 100$ bed days but has not stratified into individual medicinal compound ${ }^{8}$. The consumption of fluoroquinolones was less compared to the study in western Nepal (7.76 versus 5.43) and the finding contradicts with Shankar et al. as in our study levofloxacin was more commonly used than Ciprofloxacin ${ }^{18}$. The consumption of beta-lactam antimicrobials with beta lactamase inhibitors (ceftriaxone+tazobactam, ceftriaxone + sulbactam, Cefipime+sulbactam, Ceftazidime+sulbactam) have reduced to zero in year 2013/2014 to 2016/2017 but in the absence of ATC code, DDD could not be calculated.

The percentage of use of selected antimicrobials for prophylaxis (13\%) and empirical treatment $(43 \%)$ were found to be less compared to study in Turkey which were $44.2 \%$ and $78.4 \%$ respectively 8 . About $40.8 \%$ of aminoglycosides prescriptions were for surgical prophylaxis. The average duration of antimicrobial prophylaxis was 4.6 days which is higher than standard protocols as it recommends for no longer than $24 \mathrm{hrs}^{19,20}$.

The empiric use of antimicrobial was much less when compared to study carried out in another tertiary care hospital of Nepal where it was about $91 \%{ }^{10}$. The use of Carbapenem can be regarded significantly high if compared to study conducted in four different Hospitals in Iran as it was only 9.9 DDD/100 bed days ${ }^{21}$.

MDR organisms are bacteria that are resistant to one or more classes of antimicrobial agents recommended as first line therapy. The risk factors for infections by MDR pathogens include age, sex, previous antibiotic therapy (in the last 30 days prior to the current infection), previous hospitalization (in the last 12 months, but not in the last 3 months), patient co-morbidities and general medical condition $^{22}$. The rise in DDD value of antimicrobial such as Tigecycline, Polymyxins, Meropenem, Aztreonam implies increase in prevalence of multi drug resistant organism in this hospital as the cases of contact isolation for multi drug resistant organisms rose from 205 to 257 in 2016 to 2017 respectively and the number of patients in critical areas (Intensive care unit/High Care unit/ Cardiac Care Unit/ Pediatric Intensive Care Unit) had too 
raised from $6.8 \%$ to $7.5 \%$. Besides, unavailability of such antimicrobial agents in 2013 could too give false high result in later years. Elaborate analysis is required to justify increase in DDD/100 patient days despite restricted formulary. The use of higher grades antimicrobials mainly in critical wards supports for antimicrobial resistance as such patients are more prone to hospital acquired infection due to invasive procedures and altered physiology 23,24 .

This study does not incorporate the microbiological data to focus on use of such antimicrobial on organism specific indication. Prevalence of MDR organisms in this particular hospital to show the use of such antimicrobial could strengthen the study.

\section{Conclusions}

This study is an overview on the patterns of antimicrobials use in a tertiary care hospital of Nepal. The rise in value of DDD/100 patient beds revealed increased consumption of antibacterial over the years suggesting prevalence of multi drug organisms in this hospital. The empiric use of such higher grade anti-microbial should be discouraged by developing unit specific guidelines and measuring its compliance.

\section{Acknowledgements}

We are grateful to Dr. Subish Palain, Associate Professor of Pharmacy Practice, College of Pharmacy, Gulf Medical University, Ajman, United Arab Emirates; Dr. Anup Subedee, MD, Infectious Disease Specialist, for their help in manuscript preparation. We also thank Dr. Andrew Trotter, Assistant Professor, Division of Infectious Disease, Immunology and International Medicine, University of Illinois College of Medicine helping us design the study.

\section{References}

1. Shrikala B, Kranthi K, Nafisa A. A prospective study on evaluation of antibiotic prescription practices in an intensive care unit of a tertiary care hospital.J Clin Diagn Res.2010;4(6):338791.

2. Singh AP, Gupta U, Das S. Monitor the use of antibiotics in intensive care units with special focus on restricted antibiotics in tertiary care hospital of india. Asian J Pharm Clin Res. 2016;9: 256-59.
3. Santos EF, Lauria-Pires L, Pereira MG, Silvia AE, Rodrigues IP, Maia MO. Use of antibacterial agents in an intensive care unit in a hospital in Brazil. Braz J Infect Dis 2007;11(3):355-59.

4. Dennis C, Bergmans JJ et.al. Indications for antibiotic use in ICU patients: A oneyear prospective surveillance. J Antimicrob Chemother. 1997;39:527- 35.

5. Valizade Hasanloei MA, Sharifi H, Bushehri B, Izadseresht B. Antibiotic-use patterns in an intensive care unit of a hospital in Iran: How challenging with patient safety? Adv Biores. 2014;25-28.

6. Usluer G, Ozgunes I, Leblebicioglu H and Turkish Antibiotic Utilization Study group. A multicenter point-prevalence study: Antimicrobial prescription frequencies in hospitalized patients in Turkey. Ann Clin Microbiol Antimicrob. 2005;4:16.

7. WHO Collaborating Centre for Drug Statistics Methodology. Definition and general considerations. 2018. https://www.whocc. no/ddd/definition_and_general_considera/ Accessed 15 Dec 2017.

8. Sozen H, Gonen I, Sozen A, Kutlucan A, Kalemci S, Sahan M. Application of ATC/ DDD methodology to evaluate of antibiotic use in a general hospital in Turkey. Ann Clin Microbiol Antimicrob.2013;12:23.

9. Shankhar PR, Partha P, Dubey AK, Mishra $P$, Deshpande VY. Intensive care unit drug utilization in a teaching hospital in Nepal. Kathmandu Univ Med J. 2005;(3):130-7.

10. Shankhar PR, Partha P, Shenoy NK, Easow $\mathrm{JM}$, Brahmadathan KN. Prescribing patterns of antibiotics and sensitivity patterns of common microorganisms in the internal medicine ward of a teaching hospital in western Nepal: A prospective study. Ann Clin Microbiol Antimicrob.2003;2:7.

11. Paudel KR, Sharma M, Das BP. Prevalence of antimicrobial chemotherapy in hospitalized patients in the department of internal medicine in a tertiary care center. Nepal Med Coll J. 2008;10(2):91-5. 
12. ShankharPR,ParthaP,ShenoyN,Brahmadathan $\mathrm{KN}$. Investigation of antimicrobial use pattern in the intensive treatment unit of a teaching hospital in western Nepal. Am J infect Control. 2003; 31:410-14.

13. World Health Organization. Essential Medicines and Health products. Start using ATC/DDD. 2018. http://www.who.int/ medicines/regulation/medicines-safety/ toolkit_use/en/. Accessed 20 Dec 2017.

14. Ansari F, Erntell M, Goossens H,Davey P,ESAC II Hospital care Study Group. The European Surveillance of Antimicrobial Consumption (ESAC) point-prevalence survey of antibacterial use in 20 European hospitals in 2006. Clin Infect Dis. 2009;(49):1496-1504.

15. Luciana T, Andrajati R, Rianti A, Khan $A H$. Rational antimicrobial use in an intensive care unit in Jakarta, Indonesia: A hospitalbased, cross-sectional study. Trop J Pharm Res. 2015;14:707-14.

16. Caldeira LF, Burattini MN. Analysis of antimicrobials' consumption profile in auniversity hospital of western Paraná, Brazil. Braz J Pharm Sc. 2009;45(2):295-302.

17. Savov E, Gergova I, Borisova M, Kjoseve E, Trifonova A. Todorova I et.al. Consumption of antimicrobial drugs and antibiotic resistance in problematic for hospital infectious pathology bacteria. Trakia J Sci. 2013(4);33842.
18. Shankhar PR, Upadhyay DK, Mishra P, Palian $S$, Dubey AK, Saha AC. Fluoroquinolone utilization among inpatients in a teaching hospital in Western Nepal. J Pak Med Assoc. 2007;57(2):78-82.

19. Lerano C, Manski-Nankervis JA, Rajkhowa A, Peel T, Thursky K. Surgical antimicrobial prophylaxis. Aust Prescr.2017:225-9.

20. Bratzler DW, Dellinger EP, Olsen KM, Perl TM, et.al. Clinical practice guidelines for antimicrobial prophylaxis in surgery. Am J Health Syst Pharm.2013;70:195-283.

21. Ghaffary S, Entezari-Maleki T, Abdollohpur J, Hamishehkar H. Measurement and comparison of inpatient antibiotic use in five different hospitals in Abriz. Pharm Sci.2017; 23(1):37-41.

22. Cardoso T, Ribeiro O, Aragao IC, Costa-Peeira A, Sarmento AE. Additional risk factors for infection by multidrug-resistant pathogens in healthcare-associated infection: A large cohort study. BMC Infect Dis. 2012;12:375.

23. Trilla, A. Epidemiology of nosocomial infections in adult intensive care units. Intensive Care Med. 1994; 20(3):1-4.

24. Brusselaers N, Vogelaers D, Blot S. The rising problem of antimicrobial resistance in the intensive care unit. Ann Intensive Care. 2011;1:47. 